\title{
Projection des changements climatiques futurs
}

\author{
Olivier Boucher1, Jean-Louis Dufresne', Jessica Vial', Éric Brun², \\ Julien Cattiaux², Fabrice Chauvin2, David Salas y Mélia', \\ Aurore Voldoire ${ }^{2}$, Laurent Bopp ${ }^{3}$, Pascale Braconnot ${ }^{3}$, Philippe Ciais ${ }^{3}$, \\ Pascal Yiou ${ }^{3}$, Éric Guilyardi ${ }^{4}$, Juliette Mignot ${ }^{4}$, Céline Guivarch ${ }^{5}$ \\ 1 Laboratoire de météorologie dynamique, Institut Pierre-Simon Laplace, \\ Université Pierre-et-Marie-Curie / CNRS, Paris \\ 2 Centre national de recherches météorologiques-Groupe d'étude \\ de l'atmosphère météorologique, Météo-France / CNRS, Toulouse \\ 3 Laboratoire des sciences du climat et de l'environnement, \\ Institut Pierre-Simon Laplace, CEA / CNRS / \\ Université de Versailles Saint-Quentin-en-Yvelines, Gif-sur-Yvette \\ 4 Laboratoire d'océanographie et du climat, expérimentations \\ et approches numériques, Institut Pierre-Simon Laplace, \\ Université Pierre-et-Marie-Curie / CNRS / IRD, Paris \\ 5 Centre international de recherche sur l'environnement et le développement, \\ École des Ponts ParisTech, Nogent-sur-Marne
}

olivier.boucher@Imd.jussieu.fr

\section{Résumé}

Les modèles de climat offrent la possibilité d'anticiper la façon dont le système climatique risque d'être modifié par l'activité humaine au cours du $\mathrm{XXI}^{\mathrm{e}}$ siècle. Les études s'appuient sur des simulations numériques qui explorent l'évolution du climat moyen et de sa variabilité en fonction de différents scénarios socioéconomiques. Nous présentons une sélection de résultats du projet CMIP5 d'intercomparaison de modèles de climat avec un focus illustratif sur les deux modèles français ayant participé à cet exercice. On y décrit les effets attendus des perturbations humaines sur la température de surface, les précipitations, la cryosphère mais aussi les extrêmes météorologiques et le cycle du carbone. Les résultats font ressortir de nombreux aspects fiables, que ce soit sur l'amplitude et la répartition géographique des changements attendus ou sur les processus mis en jeu dans ces changements. Ils montrent aussi les limites de l'exercice prospectif et des incertitudes persistantes sur certains points clés.
1 serait hasardeux d'essayer de prévoir le climat futur comme une simple extrapolation des climats passés et actuel. Notre compréhension du système climatique et de ses états passés (Masson-Delmotte et al., 2015, ce numéro) démontre, s'il était nécessaire, que le climat répond aux perturbations qui s'imposent à lui. On caractérise ces perturbations en termes de forçage radiatif (Khodri et al., 2015, ce numéro), une quantité qui mesure de combien l'équilibre radiatif de la planète est modifié par une cause externe au système climatique : volcanisme, variation de l'activité solaire ou des paramètres de l'orbite terrestre, émissions anthropiques de gaz à effet de serre ou d'aérosols. S'intéresser au climat futur nécessite donc de faire des hypothèses sur l'évolution future de ces forçages que l'on a l'habitude de mesurer par rapport à la période préindustrielle (typiquement à partir de l'année 1750). Sauf éruption cataclysmique très improbable, les effets du volcanisme ne sont que transitoires et peuvent être ignorés. Par ailleurs, outre le fait qu'elles sont difficiles à prévoir, les variations de l'activité solaire ont été relativement faibles dans le passé récent; on les négligera donc dans le futur, à l'exception du cycle de 11 ans. Les variations des paramètres de l'orbite terrestre étant suffisamment lentes pour ne pas avoir d'impact significatif sur les prochains siècles, on peut donc se focaliser sur l'évolution future des forçages radiatifs d'origine anthropique dont on a vu qu'ils ont été prédominants dans l'évolution du climat du $\mathrm{XX}^{\mathrm{e}}$ siècle (Planton et al., 2015, ce numéro).

\section{Scénarios socio-économiques}

L'évolution future des émissions de gaz à effet de serre dépendra de la démographie, de l'évolution des structures socio-économiques, de l'émergence et de la diffusion de nouvelles technologies, autant de paramètres qui peuvent évoluer spontanément mais qui dépendent aussi d'éventuelles futures politiques climatiques visant à réduire ces émissions. Il est impossible de prévoir l'évolution de chacun de ces facteurs : les incertitudes sont trop grandes, du fait de l'horizon de temps très long et de la complexité des systèmes considérés. Il n'existe donc pas une " trajectoire » future d'émissions de gaz à effet de serre qu'il s'agirait de prévoir, ni même un seul monde socio-économique et technique compatible avec une trajectoire d'émission donnée. Dès lors, l'approche consiste à élaborer des 


\section{Abstract}

\section{Projection of future climate changes}

Climate models provide the opportunity to anticipate how the climate system may change due to anthropogenic activities during the $21^{\text {st }}$ century. Studies are based on numerical simulations that explore the evolution of the mean climate and its variability according to different socio-economic scenarios. We present a selection of results from phase 5 of the Climate model intercomparison project (CMIP5) with an illustrative focus on the two French models that participated to this exercise. We describe the effects of human perturbations upon surface temperature, precipitation, the cryosphere, but also extreme weather events and the carbon cycle. Results show a number of robust features, on the amplitude and geographical patterns of the expected changes and on the processes at play in these changes. They also show the limitations of such a prospective exercise and persistent uncertainties on some key aspects. scénarios socio-économiques, c'est-àdire à « raconter » plusieurs histoires des évolutions plausibles du monde. Ces histoires s'ancrent dans la connaissance des évolutions passées et cherchent à proposer des visions contrastées des futurs possibles, par exemple en opposant un monde où la globalisation continuerait de s'intensifier à un monde où une fragmentation régionale s'installerait. Ces histoires sont ensuite traduites dans des modèles, dits d'évaluation intégrée, qui décrivent les évolutions conjointes des systèmes techniques, des économies et du climat. Ces modèles permettent ainsi de quantifier les évolutions de l'agriculture, de la consommation d'énergie et donc des émissions de gaz à effet de serre dans les différentes régions du monde. Un scénario est donc la combinaison d'une " histoire », de sa traduction en valeurs des paramètres d'entrée d'un modèle socioéconomique et de la quantification des évolutions correspondantes d'un certain nombre de paramètres, comme par exemple les émissions de gaz à effet de serre, par ce modèle.

Ces trajectoires d'émissions peuvent être utilisées comme données d'entrée des modèles de climat. Ceux-ci reproduisent les circulations tridimensionnelles de l'atmosphère et de l'océan, les échanges énergétiques de la planète, le cycle hydrologique, les interactions entre le climat et les cycles biogéochimiques et offrent donc la possibilité de simuler le climat et les événements météorologiques. Les modèles sont des outils pour explorer des caractéristiques globales, régionales, voire plus locales du climat. Cet aspect exploratoire couplant scénarios et modèles de climat se traduit par une projection climatique. Une projection, à l'inverse d'une prévision, rappelle que les résultats dépendent des hypothèses formulées sur les perturbations appliquées au climat et ne reposent pas sur la connaissance des conditions initiales (voir encadré 1). Les simulations correspondantes ne se veulent donc pas forcément réalistes, mais permettent de susciter la réflexion et de comprendre comment le climat pourrait évoluer dans un contexte différent. La crédibilité des résultats vient de la compréhension de la physique et de la capacité des modèles à reproduire certaines caractéristiques clés du climat. Les scénarios socio-économiques, quant à eux, permettent à la fois : a) de coordonner les recherches sur le changement climatique en assurant la comparabilité des études qui utilisent toutes le même jeu d'émissions, b) d'explorer les incertitudes en quantifiant des visions alternatives du monde futur et c) in fine, d'éclairer les choix politiques en réponse à notre anticipation des changements climatiques (voir Guivarch et Cassen, 2015, ce numéro).

\section{Le projet CMIP5}

C'est dans cette optique qu'un nouvel ensemble de scénarios a été développé en vue du $5^{\text {e }}$ rapport d'évaluation du Groupe d'experts intergouvernemental sur l'évolution du climat (Giec) afin de

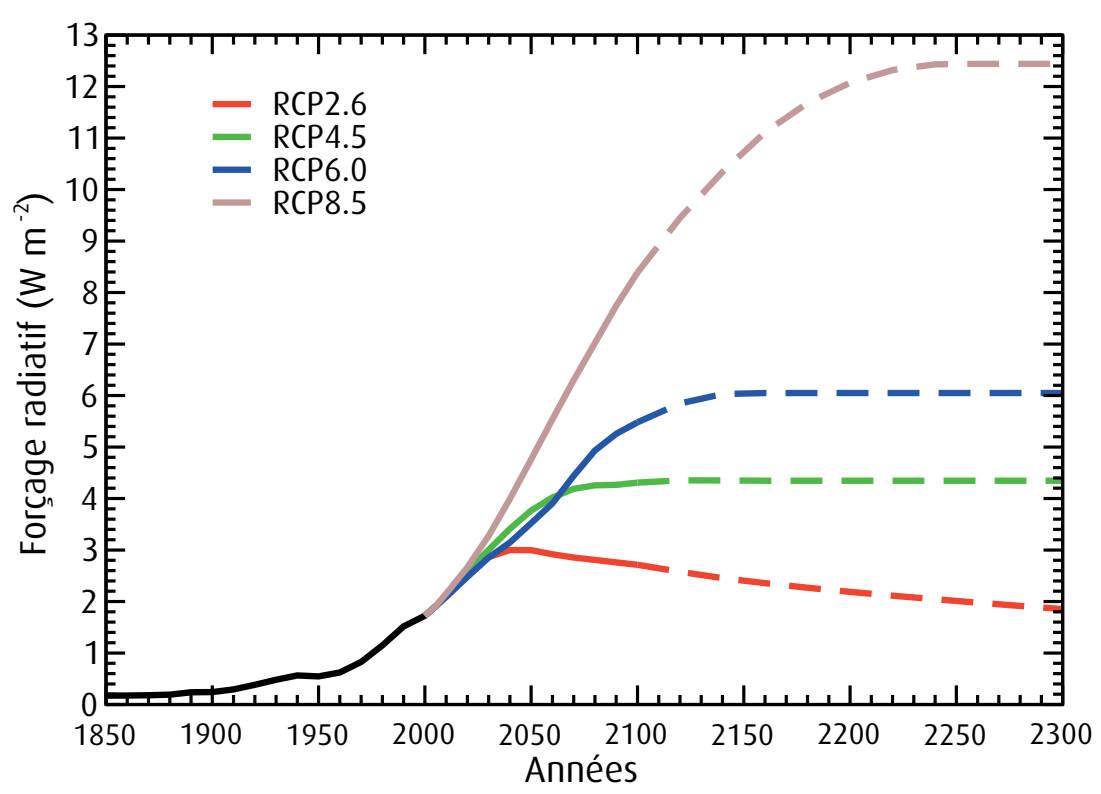

Figure 1. Évolution du forçage radiatif total et global (W m ${ }^{-2}$ ) pour la période historique (1850-2005), les quatre scénarios RCP (2005-2100) et leurs extensions jusqu'à 2300. Le forçage radiatif est exprimé par rapport à l'année de référence 1750. 


\section{La prévision à l'échelle décennale}

Le projet CMIP5 a également donné lieu à un jeu de simulations destinées à l'étude de la prévision décennale et qui ont été exploitées dans le $5^{\mathrm{e}}$ rapport du Giec (Kirtman et al., 2013). La prévision décennale a pour objet de mieux comprendre les modulations climatiques aux échelles de temps comprises entre quelques années et 30 ans dans le but de pouvoir les prévoir (Cassou et Mignot, 2013). Ces modulations sont dues à la fois à la variabilité interne du système climatique, du fait surtout de l'océan, et aux forçages dits externes tels que l'activité solaire, le volcanisme et les perturbations anthropiques. Ces recherches sont motivées par la volonté de comprendre physiquement les phénomènes, mais aussi par l'adéquation entre l'échelle de temps décennale et l'échelle de temps des décisions à prendre par la sphère socio-économico-politique dans un contexte d'adaptation au changement climatique.

Les prévisions décennales diffèrent des projections climatiques traditionnelles par l'étape d'initialisation des modèles, soit aux observations directes, soit à leurs estimations via des réanalyses. Elles cherchent en effet à reproduire la séquence temporelle de certaines des fluctuations observées, alors que les projections climatiques et simulations historiques ne visent qu'à estimer les statistiques de la réponse du système climatique aux forçages externes. En ce sens, la prévision décennale se situe à mi-chemin entre la prévision météorologique et les projections climatiques, empruntant aux deux approches leurs méthodes respectives. La première fournit le cadre nécessaire à la prise en compte des conditions initiales : procédure d'initialisation, perturbation de ces conditions initiales, réalisation d'un ensemble de plusieurs simulations (appelées membres) afin d'échantillonner au mieux le domaine des possibles et de quantifier une partie des incertitudes, et vérification à partir de simulations de prévision rétrospective. La seconde fournit le modèle du système Terre et la mise en œuvre des forçages externes. Ce pont entre ces deux champs disciplinaires a été favorisé par l'émergence de modèles de plus en plus complexes, capables de reproduire de manière satisfaisante les grandes fluctuations climatiques du passé, et l'émergence de nouveaux systèmes d'observation, en particulier les flotteurs profilants Argo qui assurent une couverture tridimensionnelle de l'océan et des produits satellitaires permettant de quantifier avec précision les échanges entre sous-systèmes climatiques.

Les premiers résultats suggèrent que la prévisibilité associée aux forçages externes est forte à l'échelle planétaire, alors que celle des modes internes dépend du bassin océanique et présente une signature régionale. Ainsi, la température de surface dans I'Atlantique Nord est plus prévisible que dans le Pacifique, avec une prévisibilité atteignant 7-8 ans dans le premier et seulement 2-3 ans dans le second. Les variables continentales (température, précipitation) sont pour l'instant peu prévisibles.

Les recherches en cours portent notamment sur la compréhension des processus, des mécanismes et rétroactions fondamentales associées aux échelles décennales, sur l'estimation et le rôle des forçages externes à ces échelles de temps, sur les méthodes de construction des conditions initiales pour le système couplé, et enfin sur l'estimation de la prévisibilité et sa vérification, véritable prérequis et défi certain au regard du peu de données disponibles. D'un point de vue scientifique, les expériences de " prévisions rétrospectives » couvrant les périodes pour lesquelles des observations sont disponibles concentrent tout autant d'intérêt que celles se focalisant sur la période future. Elles permettent en particulier de mieux comprendre les biais des modèles et leurs échelles de temps et d'espace caractéristiques.

remplacer l'ancien jeu connu sous le nom de SRES (Special report on emissions scenarios). Ce nouvel ensemble comprend quatre scénarios de référence nommés RCP2.6, RCP4.5, RCP6.0 et RCP8.5 (Moss et al., 2010 ; van Vuuren et al., 2011). Le sigle RCP est l'abréviation de Representative Concentration Pathways; il est suivi d'un nombre qui correspond à une estimation du forçage radiatif à la fin du XXI ${ }^{\mathrm{e}}$ siècle (figure 1). À la différence des SRES, les RCP sont exprimés en termes de concentrations de gaz à effet de serre et non d'émissions. Des scénarios socio-économiques très différents peuvent donc correspondre à une même trajectoire de concentrations. Pour la première fois, un scénario, le RCP2.6, inclut une politique forte de réduction des émissions de gaz à effet de serre, éventuellement accompagnée de techniques de captage $\mathrm{du} \mathrm{CO}_{2}$ atmosphérique dans la seconde moitié du siècle, afin de limiter le réchauffement climatique à $2{ }^{\circ} \mathrm{C}$ environ par rapport à la température du milieu du XIX ${ }^{\mathrm{e}}$ siècle. Le scénario RCP8.5, quant à lui, correspond à une borne haute des émissions de gaz à effet de serre et un forçage radiatif très important à la fin du XXI ${ }^{\mathrm{e}}$ siècle. Ces scénarios ont été prolongés de manière très idéalisée jusqu'en 2300 pour explorer des horizons plus lointains.
La communauté des modélisateurs du climat a organisé autour de ces scénarios une activité coordonnée de simulations climatiques communes connue sous le nom de CMIP5 (Taylor et al., 2012). Cet exercice rassemble des simulations du climat passé, des projections climatiques selon différents scénarios et des expériences plus idéalisées visant à comprendre les mécanismes du changement climatique. CMIP5 fait suite à CMIP3, dont les résultats avaient été présentés dans le $4^{\mathrm{e}}$ rapport du Giec et dans le « livre blanc Escrime » (Dufresne et al., 2006 ; Braconnot et al., 2009). La communauté française a participé à CMIP5 à l'aide des modèles CNRM-CM5.1 et IPSL-CM5A-LR (ainsi que de variantes de ce modèle) et IPSL-CM5B-LR (dont les résultats ne sont pas présentés ici). Dans la suite, on se référera simplement aux modèles du CNRM et de l'IPSL pour désigner les deux versions présentées ici. Pour la période historique et pour les quatre scénarios RCP, l'évolution de la composition chimique de l'atmosphère (concentrations d'ozone et d'aérosols en particulier) a été calculée avec les versions chimie-climat du modèle de 1'IPSL : LMDZ-Inca pour la troposphère et LMDZ-Reprobus pour la stratosphère. Les climatologies mensuelles obtenues ont été prescrites comme conditions aux limites dans les simulations du modèle de l'IPSL et après modifications dans le modèle du CNRM. Les résultats présentés dans cet article proviennent essentiellement de l'exercice CMIP5 en mettant l'accent sur les simulations du $\mathrm{XXI}^{\mathrm{e}}$ siècle réalisées avec les deux modèles de climat français. Une synthèse de ces résultats est rassemblée dans le 5e rapport d'évaluation du Giec, mais l'exploitation scientifique de ces simulations continue. Nous présentons ici quelques résultats en allant de l'échelle globale à l'échelle régionale, avant de discuter les impacts sur la cryosphère, les extrêmes météorologiques de température et de précipitations, les tempêtes et cyclones, et enfin sur le cycle du carbone. L'article se termine par une courte discussion des options d'ingénierie climatique également évoquées dans le rapport du Giec.

\section{Réchauffement global}

À partir de la simulation de contrôle qui permet de vérifier la stabilité du climat quand aucune perturbation n'est imposée, nous avons tout d'abord réalisé des simulations avec les modèles 


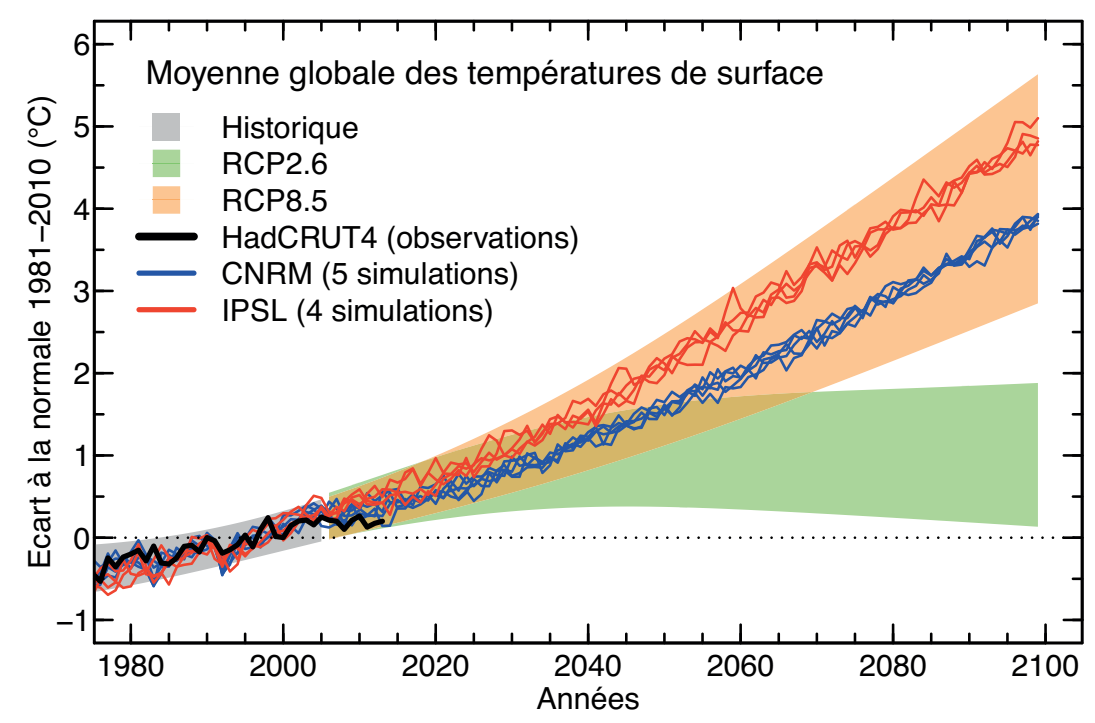

Figure 2. Écart à la normale 1981-2010 de la moyenne globale des températures de surface $\left({ }^{\circ} \mathrm{C}\right)$ pour la fin du Xxe siècle (en gris), le scénario RCP2.6 (en vert) et le scénario RCP8.5 (en orange). Les observations HadCRUT4 sont indiquées avec un trait noir et plusieurs réalisations des modèles du CNRM et de I'IPSL en scénario RCP8.5 avec des traits respectivement bleus et rouges. Les enveloppes tracées (lissées par splines) correspondent à un intervalle de confiance de $95 \%$ pour I'ensemble des modèles de climat ayant participé à l'exercice CMIP5.

Tableau 1. Réponse transitoire climatique $\left({ }^{\circ} \mathrm{C}\right)$, paramètre de sensibilité climatique à l'équilibre $\left({ }^{\circ} \mathrm{C}\right.$ $\left.\left(\mathrm{Wm}^{-2}\right)^{-1}\right)$ et paramètres de rétroaction climatique $\left(\mathrm{Wm}^{-2}{ }^{\circ} \mathrm{C}^{-1}\right)$ pour les deux modèles français ayant participé à l'exercice CMIP5 et pour la moyenne des modèles CMIP5. Selon Flato et al. (2013), sauf pour les paramètres de rétroaction du modèle CNRM-CM5.1 ( $\left.{ }^{*}\right)$ donnés par Geoffroy et al. (2014) et le paramètre de rétroaction des nuages pour les modèles CMIP5 (\$) donné par Tomassini et al. (2013). Les incertitudes données pour l'ensemble des modèles CMIP5 correspondent à des intervalles de confiance de $90 \%$. Voir l'encadré 2 pour une définition des termes utilisés. Pour le modèle de l'IPSL, le paramètre de sensibilité climatique à l'équilibre et les paramètres de rétroactions ont été diagnostiqués par différentes méthodes, ce qui explique une petite incohérence.

CNRM-CM5.1 IPSL-CM5A-LR

Moyenne des modèles CMIP5

\begin{tabular}{lccc}
\hline Réponse climatique transitoire $\left({ }^{\circ} \mathrm{C}\right)$ & 2,1 & 2,0 & $1,8 \pm 0,6$ \\
\hline $\begin{array}{l}\text { Paramètre de sensibilité climatique à l'équilibre } \\
\left({ }^{\circ} \mathrm{C}\left(\mathrm{Wm}^{-2}\right)^{-1}\right)\end{array}$ & 0,9 & 1,3 & $1,0 \pm 0,5$ \\
\hline Rétroaction de Planck $\left(\mathrm{Wm}^{-2}{ }^{\circ} \mathrm{C}^{-1}\right)$ & $-3,3^{*}$ & $-3,3$ & $-3,2 \pm 0,1$ \\
\hline Rétroaction de la vapeur d'eau $\left(\mathrm{Wm}^{-2}{ }^{\circ} \mathrm{C}-1\right)$ & $2,1^{*}$ & 1,9 & $1,6 \pm 0,3$ \\
\hline $\begin{array}{l}\text { Rétroaction du gradient vertical de température } \\
\left(\mathrm{Wm}^{-2}{ }^{\circ} \mathrm{C}^{-1}\right)\end{array}$ & $-0,5^{*}$ & $-1,0$ & $-0,6 \pm 0,4$ \\
\hline Rétroaction de l'albédo de surface $\left(\mathrm{Wm}^{-2}{ }^{\circ} \mathrm{C}-1\right)$ & $0,4^{*}$ & 0,2 & $0,3 \pm 0,1$ \\
\hline Rétroaction des nuages $\left(\mathrm{Wm}^{-2}{ }^{\circ} \mathrm{C}-1\right)$ & $0,2^{*}$ & 1,2 & $0,6(0.2-1.4) \$$ \\
\hline
\end{tabular}

climatiques en prenant en compte les différents forçages naturels et anthropiques sur la période 1850-2005. À partir de 2005, commencent les projections « futures », où les différents forçages sont imposés selon les quatre scénarios RCP présentés ci-dessus. Pour le scénario RCP8.5, les modèles simulent une élévation de la température durant tout le XXI ${ }^{\mathrm{e}}$ siècle, modulée aux plus courtes échelles de temps par la variabilité interne du climat (figure 2). L'évolution est très différente pour le scénario RCP2.6 pour lequel les modèles simulent une stabilisation progressive de la température moyenne du globe du fait d'une stabilisation puis d'une diminution des forçages (figure 1). dispersion ne s'est pas réduite depuis une vingtaine d'années. Les deux modèles français témoignent de cette incertitude avec le modèle de l'IPSL qui est plus « sensible » que celui du CNRM (tableau 1), ce qui explique les différences observées en réponse au scénario RCP8.5 (figure 2). Identifier et comprendre les raisons de cette dispersion est une des pistes pour tenter de la réduire et conduit à s'intéresser aux rétroactions climatiques. La vapeur d'eau, l'albédo des surfaces et les nuages accompagnent le changement de température découlant du forçage radiatif. Leurs modifications influencent le bilan radiatif, qui modifie en retour le changement de température (Bony et Dufresne, 2007 ; voir encadré 2). Qualitativement, la rétroaction de la vapeur d'eau amplifie la perturbation climatique initiale puisque sa concentration augmente avec la température, ce qui amplifie l'effet de serre. De même, la surface devient moins réfléchissante avec la fonte des neiges et des glaces qui accompagne le réchauffement climatique. En revanche, les rétroactions impliquant les nuages sont multiples; elles peuvent en principe être négatives ou positives.

La richesse sans précédent des expériences réalisées pour l'exercice CMIP5, notamment certaines expériences idéalisées, a permis d'améliorer, depuis le $4^{\mathrm{e}}$ rapport du Giec, la quantification des rétroactions climatiques et la compréhension des processus régissant la dispersion des estimations de sensibilité climatique entre les modèles. Encore et toujours, la principale source d'incertitude est associée aux nuages (Bony et Dufresne, 2007 ; Dufresne et Bony, 2008 ; Vial et al., 2013 ; tableau 1), qui représentent $70 \%$ de la dispersion entre les modèles sur la sensibilité climatique, avec la plus grande contribution provenant des tropiques et en particulier des régions où prédominent des nuages bas (de type stratocumulus et petits cumulus d'alizés). La boucle de rétroaction combinant les changements de vapeur d'eau et de gradient vertical de température dans les tropiques contribue substantiellement, mais en second lieu, à la dispersion de la sensibilité climatique, avec les différences entre les modèles provenant principalement des réponses de l'humidité relative dans la troposphère tropicale. La rétroaction de l'albédo de surface est relativement similaire dans les modèles. Les deux modèles de climat français sont assez symptomatiques de cet état de fait, puisqu'ils diffèrent d'abord sur la rétroaction nuageuse (1,2 


\section{Quelques définitions}

Le forçage radiatif (noté $F$ et exprimé en $\mathrm{W} \mathrm{m}^{-2}$ ) mesure le déséquilibre radiatif moyen au sommet de l'atmosphère (ou de la tropopause) induit par une perturbation climatique avant que le système climatique n'ait le temps d'y répondre.

Un scénario socio-économique peut être traduit en un scénario d'émissions de gaz à effet de serre, qui peut lui-même servir à estimer un scénario de concentrations de gaz à effet de serre. L'un ou l'autre peut servir à établir une projection climatique à l'aide d'un modèle de climat ou de système Terre.

On appelle réponse transitoire climatique le changement de la température de surface moyenne du globe $\left(\mathrm{en}{ }^{\circ} \mathrm{C}\right.$ ) au moment du doublement de la concentration en $\mathrm{CO}_{2}$ dans une expérience idéalisée où cette concentration augmente au rythme de $1 \%$ par an. Cette quantité mesure à la fois l'amplitude et la rapidité avec laquelle le système climatique répond à l'augmentation des gaz à effet de serre.

On appelle sensibilité climatique le changement de la température de surface moyenne du globe $\left(e n{ }^{\circ} \mathrm{C}\right.$ ) en réponse à un doublement de la concentration en $\mathrm{CO}_{2}$ une fois l'équilibre atteint. La sensibilité climatique est donc supérieure à la réponse transitoire climatique, mais le système peut prendre des siècles pour atteindre son nouvel équilibre. La sensibilité climatique peut être exprimée par unité de forçage radiatif, auquel cas on parle de paramètre de sensibilité climatique, sachant que la valeur canonique du forçage radiatif d'un doublement de la concentration en $\mathrm{CO}_{2}$ est égale à $3,7 \mathrm{~W} \mathrm{~m}^{-2}$. Le paramètre de sensibilité climatique est noté $\lambda$ et s'exprime en ${ }^{\circ} \mathrm{C}\left(\mathrm{W} \mathrm{m} \mathrm{m}^{-2}\right)^{-1}$.

La réponse du climat résulte d'un ensemble de rétroactions climatiques par lesquelles le changement de température de surface induit par le forçage radiatif génère une série de nouvelles perturbations radiatives dont chacune amplifie ou réduit la perturbation initiale. En premier lieu, un changement de température induit une modification du flux de rayonnement infrarouge, selon la loi de Planck, qui vient contrer le forçage radiatif. Cette rétroaction stabilisatrice très forte a un statut particulier et on lui associe souvent un paramètre de sensibilité climatique $\lambda_{0}$ qui caractériserait la réponse climatique en l'absence d'autres rétroactions. Les autres rétroactions climatiques créent des perturbations radiatives au sommet de l'atmosphère que l'on suppose proportionnelles au changement de température de surface $\Delta T$ et qui viennent s'ajouter au forçage radiatif initial $F$, si bien que le changement de température de surface peut s'écrire $\Delta T=\lambda_{0}\left(F+\Sigma_{i} C_{i} \Delta T\right)$ où les $C_{i}$ sont les paramètres de rétroaction en $\mathrm{W} \mathrm{m}^{-2}{ }^{\circ} \mathrm{C}^{-1}$ (voir tableau 1 ).

et $0,2 \mathrm{~W} \mathrm{~m}^{-2}{ }^{\circ} \mathrm{C}^{-1}$ ) et ensuite sur la rétroaction de la vapeur d'eau $(0,9$ et $1,6 \mathrm{~W} \mathrm{~m}^{-2}{ }^{\circ} \mathrm{C}^{-1}$ ).

Il a aussi été montré que la réponse de certaines variables atmosphériques dépend non seulement du changement de la température de surface mais aussi directement des variations du $\mathrm{CO}_{2}$ et des autres gaz à effet de serre qui modifient le bilan d'énergie de la troposphère, et donc sa stabilité, la formation des nuages et la précipitation, avant même que le réchauffement de la surface ne se manifeste (Risi, 2013). Cependant, les contributions de la réponse directe à l'accroissement du $\mathrm{CO}_{2}$ sur l'amplitude et l'incertitude de la sensibilité climatique sont nettement inférieures à celles des rétroactions climatiques (Vial et al., 2013). Les contributions des différentes rétroactions climatiques à la dispersion entre les modèles sur les estimations de sensibilité climatique restent d'ailleurs inchangées depuis le 4e rapport du Giec.

\section{Modifications régionales du climat}

La répartition géographique de l'accroissement de température est à peu près similaire pour les différents scénarios RCP et nous avons tracé, en figure 3 , le changement local de température de surface normalisé par le changement de température en moyenne globale. On retrouve des résultats désormais classiques : l'accroissement de température est plus élevé sur les continents que sur les océans, il est particulièrement fort dans les hautes latitudes de l'hémisphère Nord, mais moins marqué sur l'océan Atlantique nord.

Dans les régions tropicales, l'élévation de température plus importante sur les continents que sur les océans s'explique en partie par les changements d'évaporation. Sur un océan, la quantité d'eau disponible pour l'évaporation n'est pas limitée, alors que, sur un continent, la quantité d'eau qui peut s'évaporer est limitée par la quantité d'eau disponible dans le sol, et donc in fine par la quantité de précipitations. Le refroidissement induit par l'évaporation n'est donc pas limité sur les océans, alors qu'il l'est sur les continents. Dans les régions des moyennes et hautes latitudes, la faible augmentation de la température de l'océan est en partie due à son inertie thermique. Ceci est particulièrement vrai dans l'hémisphère Sud où, les vents étant très forts, l'agitation de l'océan est élevée et la température homogène sur une couche plus profonde de l'océan. Pour que la température de surface de l'océan augmente, il faut donc réchauffer une masse d'eau importante. Le minimum de réchauffement dans l'Atlantique Nord dans le modèle de l'IPSL, que l'on retrouve dans la moyenne des modèles CMIP5, est quant à lui dû à des modifications de la circulation océanique dans cette région.

Dans les hautes latitudes de l'hémisphère Nord, l'augmentation plus forte de la température est en partie due à ce que l'on appelle la rétroaction de l'albédo de surface. L'augmentation de température est accompagnée d'une diminution importante de l'enneigement et de l'extension de la glace de mer en été dans l'océan Arctique, ce qui réduit la réflexion par la surface du rayonnement solaire, augmente la quantité de rayonnement absorbé et tend à amplifier l'augmentation initiale de la température.

À l'échelle du globe, les modèles prévoient une augmentation de l'évaporation à la surface, et donc des précipitations, de l'ordre de 1 à $3 \%$ par ${ }^{\circ} \mathrm{C}$ de réchauffement (figure 6 , à gauche). Cependant, le changement de précipitations associé au réchauffement climatique est très hétérogène (figure 3). Les modèles CMIP5 s'accordent à prévoir que les hautes latitudes s'humidifient, alors qu'une grande partie des régions subtropicales s'assèche, mais les incertitudes restent fortes dès que l'on s'intéresse à l'échelle régionale. À titre d'exemple, les deux modèles français sont en désaccord sur l'amplitude, voire sur le signe, du changement annuel de précipitations pour de nombreuses régions continentales telle que l'Amazonie, l'Amérique centrale, l'Europe du Sud et la Chine. Les changements de précipitations étant largement gouvernés par des changements de circulation atmosphérique, c'est un point sur lequel devront porter les efforts futurs pour réduire ces incertitudes. 

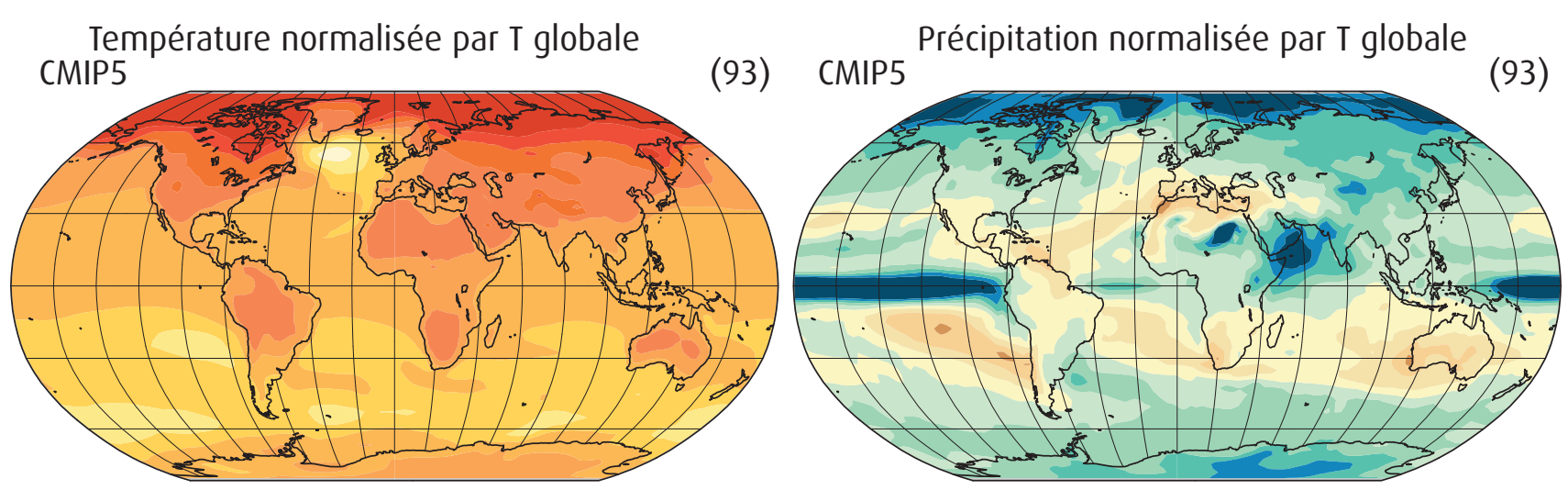

\section{CNRM}

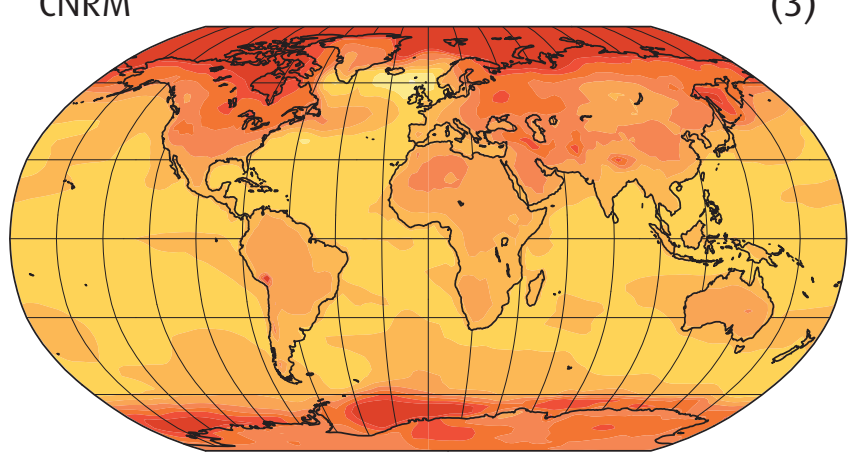

IPSL

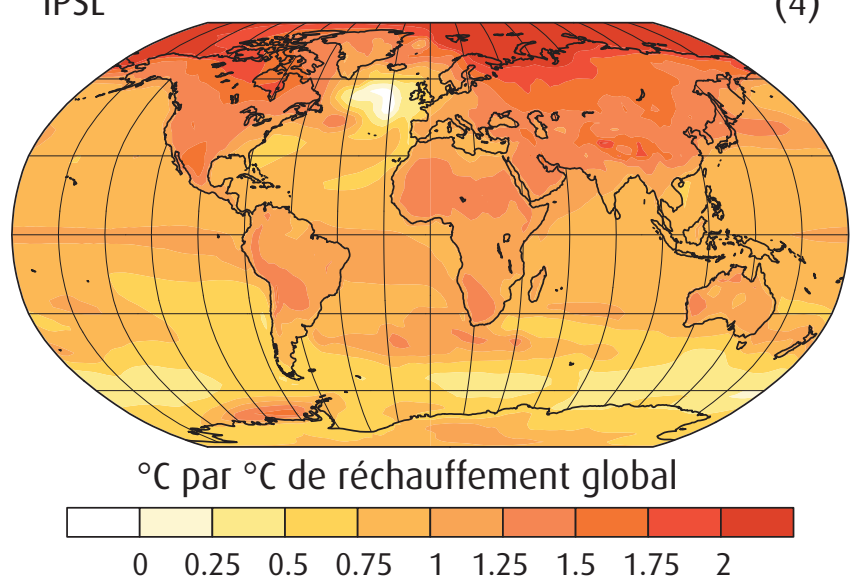

CNRM

(3)
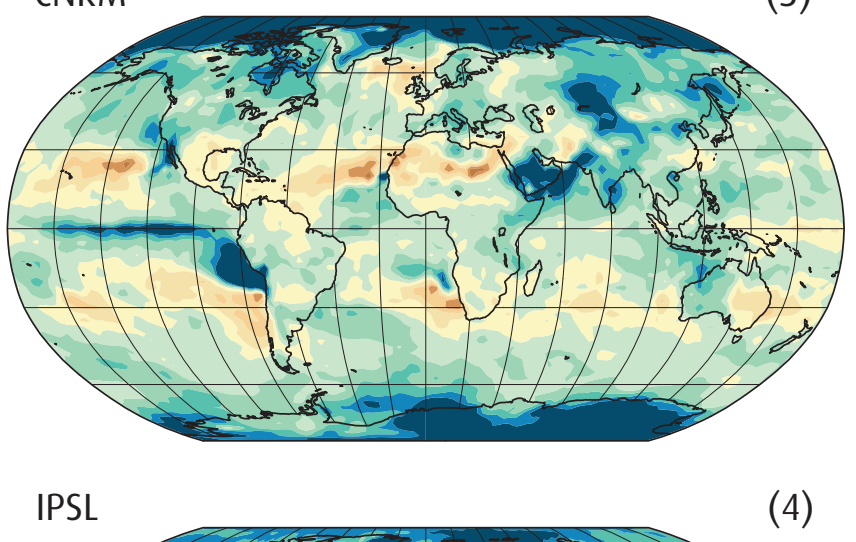

(4)

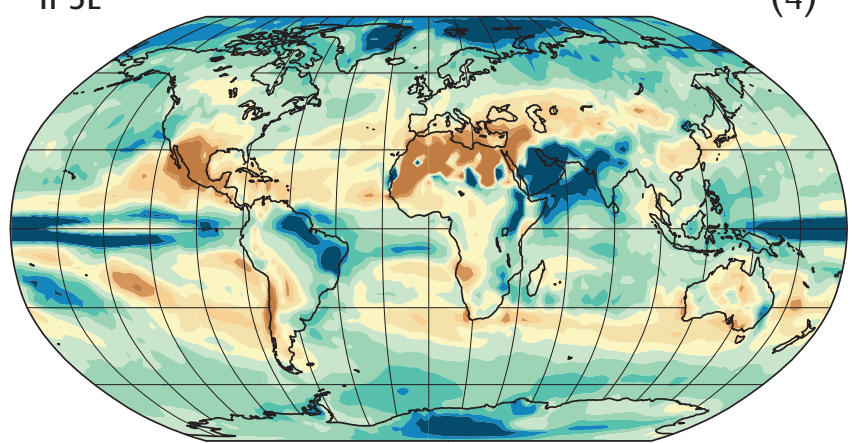

$\%$ par ${ }^{\circ} \mathrm{C}$ de réchauffement global

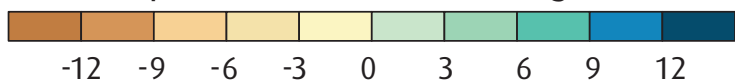

Figure 3. Changements de température (à gauche) et de précipitations (à droite) correspondant à un réchauffement global de surface de $1{ }^{\circ} \mathrm{C}$, estimés à partir de l'ensemble des modèles CMIP5 (en haut), du modèle du CNRM (au milieu) et du modèle de l'IPSL (en bas). Les cartes ont été obtenues en calculant pour chaque réalisation disponible les différences entre les périodes 2080-2099 et 1986-2005 normalisées par la valeur correspondante de réchauffement global, puis en moyennant sur toutes les réalisations (modèles et scénarios). Les nombres indiqués entre parenthèses indiquent ce nombre de réalisations pour chaque carte présentée. Les changements de température sont indiqués pour un réchauffement global moyen de $1{ }^{\circ} \mathrm{C}$ (relation linéaire). Les changements de précipitations sont indiqués en \% par degré de réchauffement global (avec une relation exponentielle, par exemple un taux de $100 \%$ par degré correspond à un quadruplement pour $\left.2^{\circ} \mathrm{C}\right)$.

\section{Évolution de la cryosphère}

Les projections du 5e rapport du Giec en termes d'évolution du couvert de neige prolongent de façon très cohérente les tendances observées durant les dernières décennies. Le signal le plus marqué est celui d'une diminution de plus en plus rapide tout au long du XXI ${ }^{\mathrm{e}}$ siècle de l'étendue de l'enneigement de l'hémisphère Nord au printemps (mars-avril), atteignant en fin de période près de $-30 \%$ de la surface actuelle pour la moyenne des modèles en scénario RCP8.5. Par contre, la masse de neige totale stockée en fin d'hiver évolue de façon incertaine sous l'effet de deux phénomènes antagonistes : d'un côté une fréquence plus faible des chutes de neige dans les régions les plus tempérées, remplacées par des pluies à cause du réchauffement, de l'autre une augmentation des précipitations neigeuses dans les régions les plus froides, à la faveur de ce même réchauffement. Dans les régions les plus froides, la plus grande épaisseur de neige en milieu d'hiver protège le sol de l'onde de gel. La plus grande précocité du déneigement printanier et un climat plus chaud s'ajoutent à cet effet pour provoquer dans la quasi-totalité des modèles une fonte accélérée du pergélisol superficiel.

Selon les projections climatiques des modèles CMIP5, la surface de la banquise arctique devrait continuer à 
diminuer. Pour le mois de septembre, c'est-à-dire le mois au cours duquel la couverture de glace est minimale, cette évolution simulée (figure 4) dépend fortement du modèle et du scénario considérés. Dans le cas du scénario RCP8.5, que ce soit pour la moyenne des modèles CMIP5, le modèle de 1'IPSL ou celui du CNRM, il est probable que l'océan Arctique soit presque entièrement dépourvu de glace ${ }^{1}$ à la fin du XXI e siècle. En revanche, dans le cas du scénario RCP2.6, la couverture de glace est moins affectée, mais son évolution dépend substantiellement du modèle considéré : par exemple, le modèle de l'IPSL simule une extension de glace proche de la moyenne d'ensemble des modèles CMIP5, tandis que le modèle du CNRM simule une extension de glace pratiquement deux fois moindre. Cependant, les modèles CMIP5 sous-estiment possiblement le recul futur de la glace, car ils le sousestiment déjà sur la période 1986-2005. Certains auteurs ont donc cherché à affiner les projections en ne retenant que les modèles CMIP5 les plus proches des observations récentes. Par exemple, Massonnet et al. (2012) ont sélectionné cinq modèles, concluant que pour quatre d'entre eux la banquise aurait pratiquement disparu avant 2050 dans le cas du scénario RCP8.5. Pour le mois de février, où l'extension de la glace de mer en Arctique est maximale, la moyenne des modèles CMIP5 indique une diminution moins prononcée de l'extension de la banquise, comprise entre $8 \%$ pour le scénario RCP2.6 et $34 \%$ pour le RCP8.5 (Collins et al., 2013).

(a)

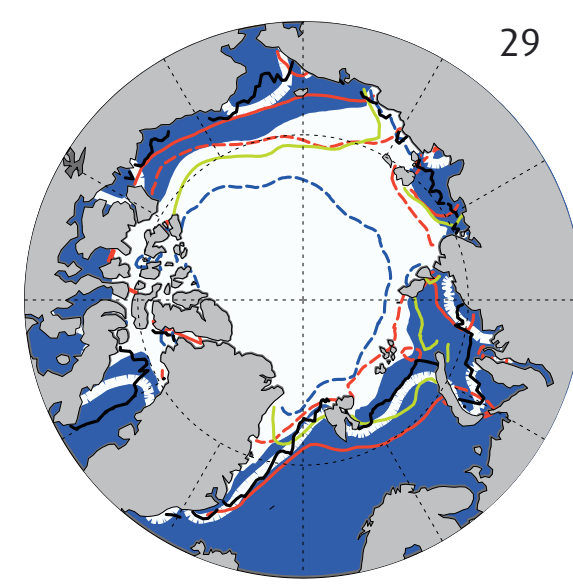

Le modèle moyen CMIP5 projette également une diminution de l'extension de la banquise antarctique. À la fin de l'hiver austral (septembre), cette diminution est de $8 \%$ (RCP2.6) et $30 \%$ (RCP8.5) pour la fin du Xxie siècle ; à la fin de l'été, l'extension minimale est réduite de 16 à $67 \%$ selon les scénarios. Cependant, le degré de confiance de ces projections est faible, car peu de modèles simulent correctement les caractéristiques observées de la banquise antarctique sur les dernières décennies, que ce soit en termes d'état moyen, de cycle saisonnier ou de tendance.

Les glaciers et les calottes glaciaires constituent une autre composante de la cryosphère très sensible au changement climatique. Leur bilan de masse n'étant pas simulé explicitement dans les modèles de l'exercice CMIP5, des analyses spécifiques ont été nécessaires pour estimer à un peu moins de $20 \mathrm{~cm}$ la contribution des glaciers et de la calotte groenlandaise à la hausse globale du niveau des mers d'ici la fin du siècle pour le scénario RCP8.5. Cela représente un peu moins de la moitié de la hausse totale attendue. L'autre moitié est liée à la dilatation thermique de la masse océanique. À cause de l'augmentation prévue des précipitations neigeuses en Antarctique, le bilan de masse superficiel augmentera, mais la fonte des glaces au contact de l'océan plus chaud augmentera également, si bien qu'il est encore impossible de conclure si la calotte antarctique contribuera à une hausse ou à une baisse du niveau des mers d'ici la fin du siècle. Une incertitude supplémentaire vient du risque de déstabilisation par un océan toujours plus chaud d'une partie des immenses plateformes glaciaires flottantes de l'Antarctique de l'Ouest, ce qui pourrait conduire à l'effondrement de tout ou partie de ces plateformes et à l'accélération des glaciers émissaires qui les alimentent.

\section{Extrêmes de température}

Au-delà du réchauffement moyen en temps ou en espace, c'est l'ensemble de la distribution des températures qui se décale vers un climat plus chaud. Ainsi, les tendances des extrêmes de température observées sur la période récente se confirment dans les projections futures : extrêmes chauds de plus en plus probables et extrêmes froids de moins en moins fréquents. Si le signe de ces tendances est robuste parmi l'ensemble des projections, leur amplitude est largement dépendante du choix du scénario d'émission et du modèle de climat, en plus de l'incertitude intrinsèque liée à la variabilité interne.
1. On considère que la banquise arctique $a$ «pratiquement disparu " lorsque son extension est inférieure à 1 million de kilomètres carrés pendant au moins cinq années consécutives.

Figure 4. Extension de la banquise arctique au mois de septembre. Les contours en trait plein sont représentatifs de la période 1986-2005. En noir : bord de glace moyen observé (données HadISST1) ; en rouge et en bleu : bords de glace simulés par les modèles de l'IPSL et du CNRM ; en blanc : bord de glace moyen simulé par l'ensemble des modèles CMIP5 pris en compte par le Giec au moment de la rédaction du rapport (le nombre de modèles utilisés est indiqué par les chiffres en haut à droite de chaque figure). Contours en traits pointillés rouge et bleu : bords de glace simulé par les modèles de l'IPSL et du CNRM en 2081-2100 pour les scénarios (a) RCP2.6 et (b) RCP8.5. Les régions représentées en blanc représentent l'extension de la banquise en 2081-2100 (moyenne des modèles CMIP5) pour les scénarios (a) RCP2.6 et (b) RCP8.5. Par convention, tous les contours représentent l'isoligne $15 \%$ de glace. Figure adaptée de la figure SPM.8c du résumé à l'intention des décideurs du 5e rapport d'évaluation du Groupe I du Giec (2013). 

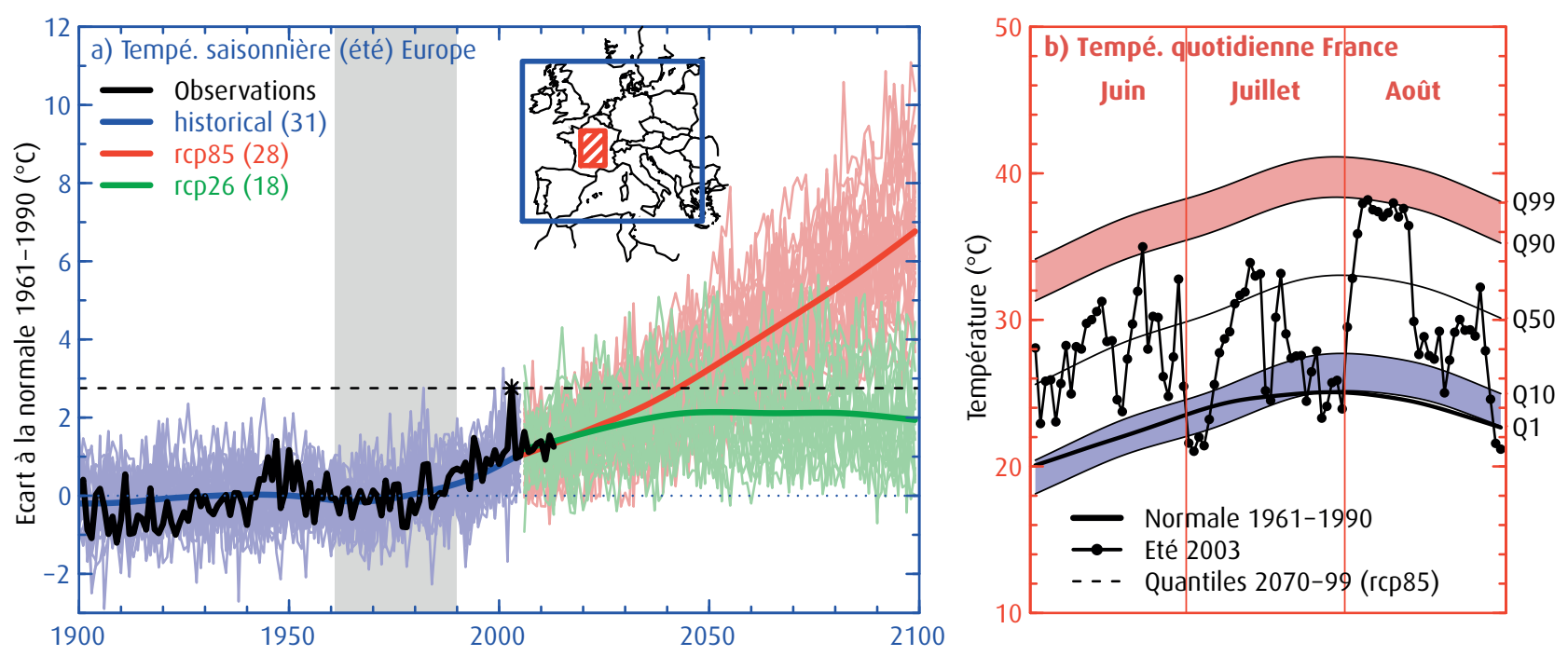

Figure 5. a) Écart à la normale 1961-1990 de la température moyennée sur l'été (juin, juillet, août) et l'Europe (boîte bleue sur la carte) pour les observations (CRUTEM4v, trait noir), les simulations historiques individuelles (traits bleus) et les projections futures en scénarios RCP8.5 (traits rouges) et RCP2.6 (traits verts). Le nombre de simulations utilisées ainsi que les moyennes d'ensemble lissées (traits épais avec couleurs correspondantes) sont indiqués. L'anomalie de $2003\left(2,8^{\circ} \mathrm{C}\right.$, marquée par une étoile) devient froide en RCP8.5 dès 2050, mais reste chaude en RCP2.6 jusqu'en 2100. b) Température quotidienne de l'été 2003 (juin, juillet, août) moyennée sur le centre de la France (boîte hachurée rouge sur la carte), comparée à la normale observée (E-OBS, noir épais) et à la moyenne des quantiles 2070-2099 sur toutes les simulations RCP8.5 (bleu entre 01 et 010, rouge entre 090 et 099, voir échelle de droite).

En moyenne sur les modèles, et aux échelles globale et annuelle, la probabilité d'observer une température quotidienne supérieure au $90^{\mathrm{e}}$ centile actuel passe ainsi de $10 \%$ (un jour sur dix, par définition) à $25 \%$ (un jour sur quatre) en scénario RCP2.6, voire à $60 \%$ (plus d'un jour sur deux) en scénario RCP8.5, d'ici 2100 (Sillmann et al., 2013). À l'inverse, la fréquence de jours froids

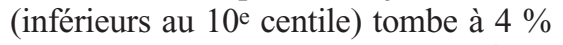
en RCP2.6 et à $1 \%$ en RCP8.5. Cela se répercute sur les événements rares : par exemple, un événement chaud de période de retour de 20 ans en climat actuel se produit en moyenne une année sur deux vers 2100 en RCP8.5, tandis que son symétrique froid voit sa période de retour dépasser le siècle (Kharin et al., 2013). Malgré ces tendances, la variabilité interne rend la survenue d'extrêmes froids ponctuels toujours possible, même dans un scénario de réchauffement élevé, surtout dans les régions où cette variabilité est forte (Kodra et al., 2011). Les records de froid seront moins fréquents que les records de chaleur au $\mathrm{XXI}^{\mathrm{e}}$ siècle. Par exemple, le ratio, évalué à deux records de chaleur pour un record de froid (2/1) en climat actuel sur les États-Unis, atteint 20/1 autour de 2050 et 50/1 vers 2100 pour un scénario de réchauffement modéré (Meehl et al., 2009).

En plus des incertitudes liées au choix du scénario et à la variabilité interne, l'évolution future des extrêmes de température est dépendante de la sensibilité du modèle de climat, en particulier aux échelles régionales et saisonnières. En Europe et en été, la probabilité de dépasser le 90e centile actuel de température quotidienne passe ainsi de $10 \%$ (c'est-à-dire un jour sur dix, par définition) à 30 à $90 \%$ selon le modèle en fin de $\mathrm{XXI}^{\mathrm{e}}$ siècle pour le scénario RCP8.5 (respectivement $50 \%$ et $80 \%$ pour les modèles du CNRM et de 1'IPSL; Cattiaux et al., 2013). Ces incertitudes se reportent sur les caractéristiques des événements plurijournaliers. Par exemple, bien que les projections futures s'accordent sur l'augmentation de la fréquence, de l'intensité et de la durée des canicules estivales européennes, l'amplitude de leur évolution d'ici 2100 peut varier du simple au triple selon le modèle et le scénario considérés (Schoetter et al., 2014). Enfin, si les changements dans les extrêmes de température sont liés en premier lieu à l'amplitude du réchauffement moyen (décalage de la distribution), ils sont aussi modulés par les changements de variabilité (forme de la distribution). En Europe, les projections futures suggèrent une augmentation de la variabilité en été et une réduction en hiver, respectivement liées à un assèchement des sols et une diminution de la couverture neigeuse.

L'été caniculaire de 2003 est-il un prototype des étés européens du $\mathrm{XXI}^{\mathrm{e}}$ siècle ? À l'échelle de l'Europe de l'Ouest et de la saison entière, avec une anomalie avoisinant les $3{ }^{\circ} \mathrm{C}$ par rapport aux normales 1961-1990, il rejoint la moyenne des projections RCP8.5 dès la décennie 2040, devenant même un extrême froid en 2100 (figure 5a). Au plus fort des canicules de juin et août, les températures exceptionnelles de 2003 rejoignent le 90e centile de la distribution simulée en fin de $\mathrm{XXI}^{\mathrm{e}}$ siècle. L'été 2003 demeure cependant un été anormalement chaud jusqu'en 2100 dans le scénario RCP2.6. Par ailleurs, à une échelle de quelques jours et locale (en France), les jours les plus chauds d'août 2003 restent anormalement chauds même en 2100 en scénario $\mathrm{RCP8.5}$ (figure $5 \mathrm{~b}$ ). La réponse à cette question est donc à moduler selon le scénario et l'échelle spatio-temporelle considérés.

\section{Extrêmes hydrologiques}

Alors que la réponse des extrema de température aux forçages anthropiques s'explique en bonne partie par une simple translation de la distribution des températures quotidiennes par le réchauffement simulé en moyenne saisonnière, la réponse des précipitations horaires ou quotidiennes est plus complexe et peut se traduire par une modification significative des moments d'ordre supérieur de la distribution. Ainsi, dans la plupart des régions, on peut s'attendre à un renforcement des pluies les plus intenses au cours du $\mathrm{XXI}^{\mathrm{e}}$ siècle (et de leur contribution aux précipitations totales). Bien que, pour 
des raisons énergétiques (O'Gorman et al., 2012 ; Boucher et al., 2013), la réponse des précipitations globales aux forçages anthropiques soit très en deçà (qui indique que le contenu maximal en vapeur d'eau de l'atmosphère augmente d'environ $7 \%$ par degré de réchauffement), la question est de savoir si, concernant les événements extrêmes en particulier, la sensibilité des précipitations n'est pas nettement plus élevée, Clapeyron (O’Gorman, 2012). Lorsque les conditions synoptiques sont réunies pour mobiliser l'essentiel de la vapeur d'eau que peut contenir l'atmosphère (tempêtes et cyclones, mais également orages plus ou moins isolés), la théorie prévoit que le réchauffement global devrait conduire à une nette augmentation des précipitations associées (Trenberth, 2011). C'est aussi ce que prévoient la plupart des modèles et ce que l'on commence à détecter dans les séries d'observations (figure 6). La résolution limitée des modèles et leur difficulté à reproduire l'amplitude de la variabilité multidécennale des précipitations observées laissent toutefois craindre que ce risque puisse être encore sous-estimé. On notera aussi que de nouveaux travaux indiquent que les chutes de neige extrêmes seraient relativement insensibles au réchauffement climatique (O’Gorman, 2014). de la relation de Clausius-Clapeyron voire au-delà de la relation de Clausius-

Le nombre de jours sans pluie devrait également globalement augmenter. Le fait que les précipitations globales augmentent à un taux moindre que la vapeur d'eau implique en effet un ralentissement du cycle hydrologique et une raréfaction des pluies, notamment dans les régions et aux saisons où celles-ci sont déjà peu abondantes. C'est ce qui fait dire à certains qu'en termes de précipitations, les riches deviendront plus riches et les pauvres plus pauvres («the rich get richer and the poor get poorer »), même si ce principe ne peut pas être généralisé à toutes les régions continentales. Cette possibilité est particulièrement alarmante pour l'évolution des risques de sécheresse, d'autant que le réchauffement global peut également se traduire par un accroissement de la demande évaporative et donc de l'évaporation effective dès que l'humidité du sol n'est pas le facteur limitant. L'effet de l'homme sur l'évapotranspiration des surfaces continentales a d'ores et déjà été mis en évidence (Douville et $a l ., 2013$ ) et la poursuite des émissions de gaz à effet de serre ainsi que le déclin des émissions des aérosols (et de leur effet refroidissant) dans certaines régions du globe devraient conduire à renforcer encore cette demande évaporative (Gedney et al., 2014), laissant craindre une augmentation du nombre, de la durée, de l'intensité et de l'extension des sécheresses. Si l'on ajoute à

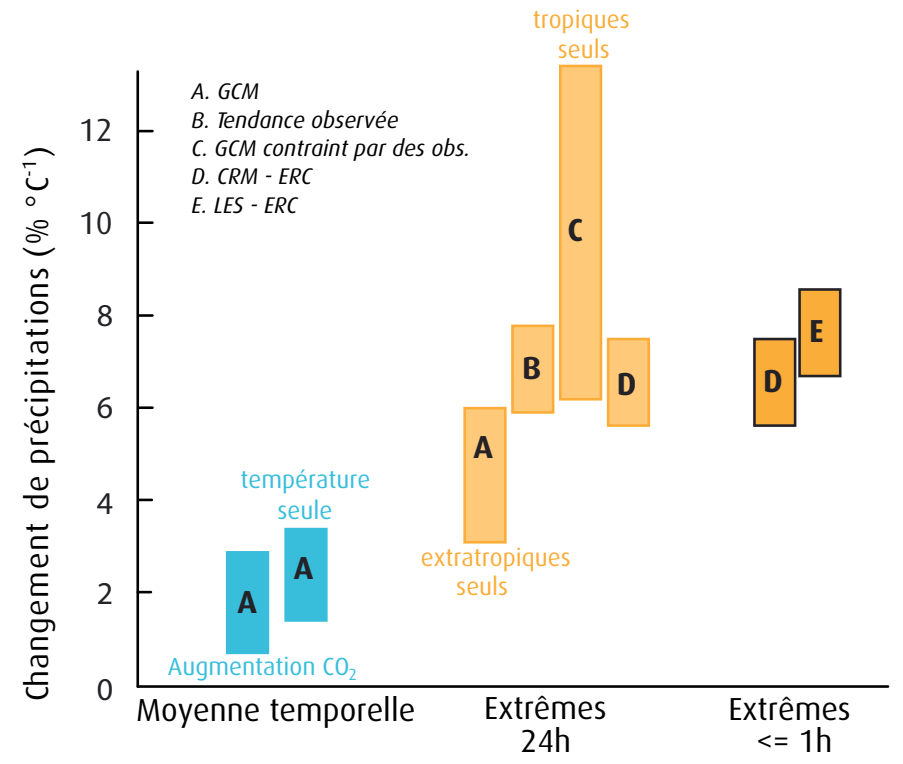

Figure 6. Estimation de l'augmentation des quantités de précipitations par degré de réchauffement global. À gauche, en bleu, augmentation de la précipitation à l'échelle globale avec ou sans la réponse directe à l'accroissement du $\mathrm{CO}_{2}$ atmosphérique qui contribue à stabiliser l'atmosphère. Au centre, en orange clair, augmentation du 99,9e centile du total journalier de la précipitation à l'échelle locale. A droite, en orange foncé, augmentation du 99,9e centile du total horaire de la précipitation à l'échelle locale. On distingue les estimations selon qu'elles proviennent des modèles de climat (A), des longues séries d'observations de surface (B), des modèles de climat corrigés par des observations des extrêmes (C) et des modèles de petite échelle de type Cloud Resolving Model (CRM) ou Large Eddy Simulations (LES) (D, E). Les barres verticales correspondent à un intervalle de confiance de $90 \%$. Adapté de Boucher et al. (2013). cela que l'intensification des pluies peut se traduire par une baisse de l'infiltration au profit du ruissellement en surface, on réalise à quel point la gestion des ressources en eau sera un problème de plus en plus aigu dans de nombreuses régions, et en particulier sur le pourtour du bassin méditerranéen. On notera finalement que, comme pour les extrêmes en température, les changements d'extrêmes hydrologiques dépendent fortement $\mathrm{du}$ scénario considéré.

\section{Fréquence et intensité des tempêtes des moyennes latitudes}

Alors que le $4 \mathrm{e}$ rapport du Giec semblait indiquer un certain consensus entre les modèles ayant participé à l'exercice CMIP3 quant au décalage vers les pôles de l'activité synoptique future, les dernières simulations CMIP5 tendent à relativiser ce résultat. Ce comportement peut s'expliquer par le fait que l'activité synoptique répond à de nombreux forçages dont les évolutions futures peuvent entrer en compétition. Le gradient méridien de température, qui est la principale source d'énergie des dépressions de moyenne latitude, devrait s'estomper dans les basses couches de la troposphère, alors qu'il devrait s'accroître en altitude, ce qui produirait un effet différencié entre ces deux niveaux. De même, le changement de température avec l'altitude devrait se trouver perturbé avec une augmentation de l'instabilité vers les pôles et une diminution vers l'équateur. De plus, l'évolution même de l'activité synoptique peut moduler la manière dont le climat répond aux forçages anthropiques, ce qui rend la tâche des modèles encore plus compliquée. Enfin, si l'hémisphère Sud semble faire 1'objet d'un large consensus entre les modèles quant à une diminution de l'activité synoptique associée à un décalage vers le pôle Sud, les réponses sont moins marquées dans l'hémisphère Nord, en partie à cause d'une couverture beaucoup plus étendue de ses continents. Un signal comparable à celui de l'hémisphère Sud semble émerger pour le bassin Pacifique, alors que celui de l'Atlantique est plus complexe, avec une baisse d'activité dans le sud-ouest du bassin et une augmentation au large de l'Europe du Nord. Un signal clair, néanmoins, émerge sur la Méditerranée, avec une baisse significative des dépressions. 
À plus brève échéance, c'est-à-dire pour les trente prochaines années, les signaux indiqués par les modèles ne sortent pas de la variabilité naturelle de l'activité synoptique, elle-même fortement corrélée avec celle des grands modes de variabilité atmosphérique (oscillation arctique et nord-atlantique) et océanique (oscillation atlantique multidécennale). Le décalage vers le pôle de l'activité de l'hémisphère Sud devrait quant à lui être nettement moins évident que pour la fin du siècle. En effet, l'activité synoptique est liée au principal mode de variabilité de l'hémisphère Sud (le mode annulaire) qui luimême dépend de la concentration d'ozone stratosphérique. Le comblement du trou d'ozone, dont l'achèvement n'est pas prévu avant les années 2060-2070, tend à imprimer un décalage vers l'équateur de l'activité synoptique, en opposition avec le signal lié à la concentration des gaz à effet de serre.

\section{Fréquence et intensité des cyclones}

L'utilisation de la base de données CMIP5 pour les études liées à l'activité cyclonique est limitée par la résolution des simulations qui la composent. Audessus de $100 \mathrm{~km}$ de résolution, la représentation des cyclones tropicaux est trop grossière pour espérer interpréter des changements dans les trajectoires ou même les caractéristiques des cyclones. Ces simulations permettent tout au plus d'étudier les tendances dans des indices de cyclogenèse fondés sur les conditions de grande échelle favorables au développement de systèmes cycloniques. Les rares simulations globales dont la résolution est inférieure à $50 \mathrm{~km}$ permettent de détecter directement les systèmes cycloniques et de les suivre dans leur cycle de vie. Selon ces simulations, le nombre de cyclones au cours du XXI siècle ne devrait pas changer ou être en légère baisse. En revanche, la probabilité de voir apparaître des systèmes de forte intensité devrait augmenter avec le réchauffement. De même, les simulations s'accordent sur une augmentation substantielle des pluies associées aux cyclones. De nouvelles interrogations ont néanmoins vu le jour dans le dernier rapport du Giec. L'évolution des grands modes de variabilité du climat et leur sensibilité aux activités humaines restent incertaines, alors que ceux-ci ont un fort impact sur l'activité cyclonique, notamment audessus du bassin atlantique. Par ailleurs, plus que l'amplitude du réchauffement des températures de la mer, c'est sa structure spatiale qui semble primordiale pour comprendre l'évolution future de l'activité cyclonique. Ces deux points figurent parmi les grandes incertitudes du climat à venir.

\section{Et le cycle du carbone?}

Le cycle global du carbone contrôle en grande partie l'évolution future du climat. Sur les 40 dernières années, environ la moitié des émissions anthropiques de $\mathrm{CO}_{2}$ (dues aux émissions de carbone fossile et à la déforestation) a été absorbée, à parts à peu près égales, par l'océan et la biosphère terrestre. Ces puits de carbone naturels sont liés à des processus physiques, chimiques et biologiques qui diffèrent largement entre régions et varient dans le temps. Le puits océanique est dû à la différence de pression partielle entre le $\mathrm{CO}_{2}$ atmosphérique (qui augmente en raison des émissions anthropiques) et le $\mathrm{CO}_{2}$ dissous à la surface des océans, qui dépend lui-même du mélange physique entre océan superficiel et couches d'eaux sous-jacentes, de l'équilibre chimique entre $\mathrm{CO}_{2}$ dissous, ions bicarbonates et carbonates, et de l'activité biologique qui peut consommer du carbone (via la photosynthèse) ou en produire (via la reminéralisation de la matière organique).

Le puits de carbone dans la biosphère terrestre est contrôlé par des processus tout aussi complexes comme la réponse de la photosynthèse à l'augmentation du $\mathrm{CO}_{2}$ et au changement climatique, la réponse de la mortalité des plantes et de la décomposition de la litière et du carbone du sol en réponse à des changements du climat et de la photosynthèse. Les flux de carbone anthropiques dans la biosphère continentale dépendent en outre des quantités de nutriments disponibles, principalement azote et phosphore, eux-mêmes perturbés par l'activité humaine (apports d'engrais sur les écosystèmes cultivés, dépôts atmosphériques d'oxydes d'azote...). Enfin, la gestion des écosystèmes par l'homme, comme les prairies et les cultures mais aussi les forêts exploitées, a un fort impact sur leur capacité à stocker du carbone, car elle modifie les apports en nutriments, le temps de résidence du carbone dans la biomasse et les sols, et provoque, dans le cas de la déforestation et des feux anthropiques, une émission directe de $\mathrm{CO}_{2}$ vers l'atmosphère.
Les flux de carbone répondent aux changements de $\mathrm{CO}_{2}$ atmosphérique et du climat, et aux changements d'apports en nutriments. En retour, les puits de $\mathrm{CO}_{2}$ pour un scénario d'émission donné influencent la fraction des émissions de carbone qui s'accumule dans l'atmosphère et donc le forçage radiatif du $\mathrm{CO}_{2}$. Les premiers modèles couplés tridimensionnels du climat et du cycle du carbone, précurseurs des modèles de système Terre qui considèrent aussi les interactions avec la chimie atmosphérique et les aérosols, ont été produits au début des années 2000 et ont été considérés pour la première fois par le Giec dans son $4 \mathrm{e}$ rapport d'évaluation. Leurs résultats montrent une rétroaction positive entre climat et cycle du carbone, c'est-à-dire une amplification du réchauffement provoquée par l'affaiblissement des puits de $\mathrm{CO}_{2}$ dans l'océan et la biosphère continentale. Cet affaiblissement des puits s'explique, pour l'océan, par le réchauffement des eaux de surface qui diminue la solubilité du $\mathrm{CO}_{2}$ et par la stratification verticale de l'océan qui limite la pénétration du $\mathrm{CO}_{2}$ anthropique dans les couches profondes. Pour la biosphère terrestre, ce sont principalement les forêts tropicales qui sont responsables de l'affaiblissement du puits, car elles répondent à une baisse des précipitations. Toutefois, les modèles donnent des amplitudes très différentes pour cette rétroaction, qui est en fait la résultante d'une rétroaction négative (stabilisation) liée à l'augmentation de la concentration atmosphérique du $\mathrm{CO}_{2}$ qui renforce les puits, et d'une rétroaction positive (amplification) liée aux effets du réchauffement climatique. Les résultats présentés dans le $5^{\mathrm{e}}$ rapport d'évaluation (Ciais et al., 2013) sont fondés sur près d'une dizaine de modèles couplés climat-carbone. Ils confirment l'existence de la rétroaction positive, mais l'incertitude n'a pas été réduite significativement par rapport au $4^{\mathrm{e}}$ rapport d'évaluation. Un seul modèle inclut les interactions entre les cycles de l'azote et du carbone dans la biosphère terrestre, et aucun modèle n'inclut les limitations par le phosphore du sol.

Dans le $5^{\mathrm{e}}$ rapport du Giec (Ciais et al., 2013), les modèles couplés climatcarbone, qui simulent de façon conjointe climat et cycle du carbone, ont été utilisés pour traduire les trajectoires futures de concentration du $\mathrm{CO}_{2}$ atmosphérique (prescrites à partir des Representative Concentration Pathways évoqués ci-dessus) en émissions anthropiques « compatibles ». En effet, en utilisant les flux de carbone calculés par 


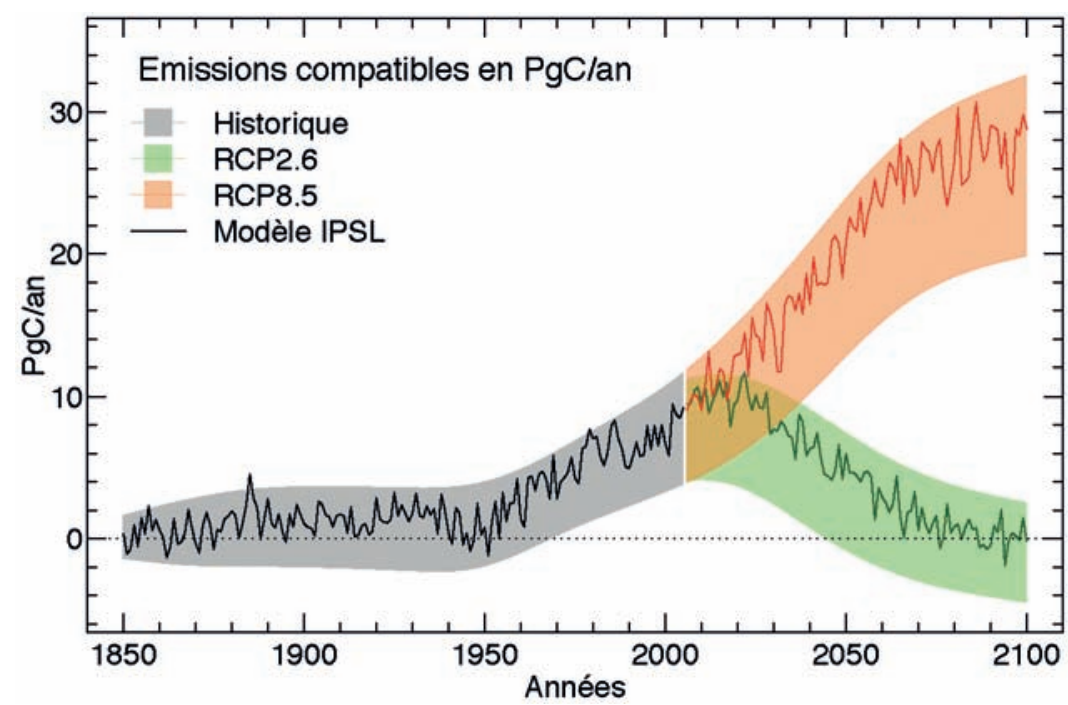

Figure 7. Émissions de $\mathrm{CO}_{2}$ compatibles (en $\mathrm{Pg} \mathrm{C}$ par an ou milliards de tonnes de carbone par an) estimées dans les modèles à partir des puits de carbone (océaniques et continentaux) simulés et des trajectoires de concentration de $\mathrm{CO}_{2}$ atmosphérique imposées (voir texte) pour la période historique (en gris) et les scénarios RCP2.6 (en vert) et RCP8.5 (en orange). Le modèle de l'IPSL est représenté par un trait plein et l'enveloppe représente l'intervalle entre les $2,5^{\mathrm{e}}$ et $97,5^{\mathrm{e}}$ centiles dans l'ensemble CMIP5 (limité à 10 modèles) en faisant l'hypothèse d'une répartition gaussienne de leur résultats.

les modèles entre l'atmosphère et les puits de carbone (océan, biosphère terrestre), on peut calculer la quantité de carbone qu'il faudrait émettre chaque année pour suivre une trajectoire de $\mathrm{CO}_{2}$ atmosphérique donnée. Ces estimations ont permis de préciser les quantités de carbone que l'on pourrait émettre pour rester sur les trajectoires des différents scénarios RCP (figure 7). À partir des résultats d'une dizaine de modèles, le Giec conclut que ces émissions totales compatibles pour la période 2012-2100 s'élèvent respectivement entre 140 et 410 , entre 595 et 1005 , entre 840 et 1250 , et entre 1415 et 1910 milliards de tonnes de carbone pour les trajectoires RCP2.6, RCP4.5, RCP6.0 et RCP8.5.

\section{Un mot sur l'ingénierie climatique}

La perspective d'un changement climatique élevé d'ici à 2100 si les émissions de gaz à effet de serre continuent à progresser a conduit certains ingénieurs et chercheurs à imaginer comment modifier le climat de manière intentionnelle afin de limiter le changement climatique ou certains de ses impacts (Boucher, 2012). L'exercice CMIP5 ne considère pas explicitement de scénarios de l'ingénierie climatique, même si le scénario RCP2.6 implique des émissions négatives de $\mathrm{CO}_{2}$ à partir de 2050 pour un certain nombre de modèles.
Cependant, des simulations climatiques ont été réalisées dans le cadre de l'exercice GeoMIP afin d'étudier le potentiel, les limitations et les risques des techniques de « gestion du rayonnement solaire », comme l'injection d'aérosols dans la stratosphère ou encore l'injection d'embruns marins au-dessus des océans dans le but d'augmenter la réflectivité des nuages (Kravitz et al., 2011). Sans préjuger de la faisabilité technique ou économique de ces techniques, les modèles de climat s'accordent à dire que le potentiel de refroidissement de la planète est réel, tout du moins pour la technique d'injection d'aérosols stratosphériques, la modification de la réflectivité des nuages étant beaucoup plus incertaine. Cependant, des limitations et de nombreux risques ont été mis en évidence. Il est démontré que le refroidissement induit par l'augmentation de l'albédo de la planète ne peut compenser parfaitement le réchauffement dû à l'augmentation des gaz à effet de serre. En particulier, on ne peut maintenir à la fois température et précipitation inchangées en moyenne globale, et encore moins à l'échelle régionale. La gestion du rayonnement solaire impliquerait donc des changements de précipitation qui, à l'échelle régionale, pourraient être aussi néfastes que ceux induits par le réchauffement climatique que l'on chercherait à contrer. L'injection d'aérosols dans la stratosphère présente aussi des risques pour la couche d'ozone et aucune des techniques de gestion du rayonnement solaire ne s'attaque à la cause du réchauffement climatique et ne résout le problème d'acidification des océans. Les modèles montrent aussi que si les émissions d'aérosols (dans la stratosphère ou dans la couche limite marine) sont interrompues pour une raison quelconque, alors même que les concentrations de gaz à effet de serre auraient continué d'augmenter, la planète connaîtrait un « rattrapage climatique » à l'échelle d'une décennie, avec un taux de réchauffement plus élevé et une adaptation au changement climatique encore plus difficile que s'il n'y avait pas eu de d'ingénierie climatique. Ces techniques sont donc problématiques à plus d'un titre mais continuent néanmoins d'être étudiées.

\section{Conclusions}

L'exercice CMIP5 et le $5^{\text {e }}$ rapport d'évaluation du Giec qui s'en est suivi ont confirmé bon nombre des résultats obtenus avec CMIP3 et les conclusions $\mathrm{du} 4^{\mathrm{e}}$ rapport d'évaluation. Cela montre la robustesse des grands traits du réchauffement climatique attendu pour le $\mathrm{XXI}^{\mathrm{e}}$ siècle : réchauffement amplifié au-dessus des continents et des hautes latitudes de l'hémisphère Nord, variations différenciées des précipitations, augmentation des extrêmes chauds de température, mais aussi des extrêmes de précipitations dans de nombreuses régions, diminution de l'enneigement et fonte de la glace de mer dans l'Arctique, diminution de la fraction de $\mathrm{CO}_{2}$ absorbée par la végétation et l'océan ou encore élévation du niveau des mers (Cazenave et al., 2015, ce numéro). Cependant, des incertitudes importantes résistent aux efforts des chercheurs, concernant à la fois le réchauffement moyen et les aspects régionaux du changement climatique. Les intervalles de confiance donnés par le Giec restent larges, aussi bien pour la réponse climatique transitoire (de 1 à $2,5^{\circ} \mathrm{C}$ ) que pour la sensibilité climatique (de 1,5 à $4,5^{\circ} \mathrm{C}$ ), avec des implications importantes en termes d'impacts et d'émissions de $\mathrm{CO}_{2}$ compatibles avec un objectif climatique donné. Une grande partie de ces incertitudes provient de la rétroaction nuageuse qui, même si elle est de mieux en mieux caractérisée et comprise dans les modèles, n'en reste pas moins particulièrement incertaine. On se heurte ici à l'absence de contraintes observationnelles fortes sur la sensibilité climatique, qu'elles soient directes via la connaissance conjointe du changement de température de surface et des forçages 
radiatifs ou indirectes via les rétroactions. Au final, et compte tenu de ces incertitudes, seul le scénario RCP2.6, qui implique des émissions négatives de gaz à effet de serre à partir de 2050 dans certains modèles, permet de rester sous le seuil des $2{ }^{\circ} \mathrm{C}$ de réchauffement par rapport à la période pré-industrielle avec une probabilité élevée.

\section{Remerciements}

Nous remercions tous les collègues qui ont contribué au développement des versions des modèles de l'IPSL et du CNRM, et à la réalisation des nombreuses expériences de l'exercice CMIP5, sans lesquels cet article n'aurait pu voir le jour. Le modèle CNRM-CM5.1 a été développé conjointement par le CNRM et le Cerfacs. Les collaborations nationales se font au travers du projet Missterre financé par le programme national interorganisme LEFE.

\section{Bibliographie}

Bony S., Dufresne J.-L., 2007. Processus régissant la sensibilité climatique. La Météorologie, 56, 29-32.

Boucher 0., 2012. L'ingénierie climatique : solution d'avenir ou fuite en avant au problème du changement climatique? La Météorologie, 78, 31-43.

Boucher O., Randall D., Artaxo P., Bretherton C., Feingold G., Forster P., Kerminen V.-M., Kondo Y., Liao H., Lohmann U., Rasch P., Satheesh S.K., Sherwood S., Stevens B., Zhang, X.Y., 2013. Clouds and aerosols, In: Climate Change 2013: The Physical Science Basis. Contribution of Working Group I to the Fifth Assessment Report of the Intergovernmental Panel on Climate Change (Stocker T.F., Qin D., Plattner G.-K., Tignor M., Allen S.K., Boschung J., Nauels A., Xia Y., Bex V., Midgley P.M., eds). Cambridge University Press, Cambridge, UK, New York, NY, USA.

Braconnot P., Dufresne J.-L., Salas y Mélia D., Terray L., 2009. Analyse et modélisation du changement climatique. 2e édition du Livre blanc Escrime. Société Météorologique de France.

Cassou C., Mignot J., 2013. Enjeux, méthodes et fondamentaux de prévisibilité et prévision décennale. La Météorologie, 81, 23-30.

Cattiaux J., Douville H., Peings Y., 2013. European temperatures in CMIP5: Origins of present-day biases and future uncertainties. Clim. Dyn., 41, 2889-2907.

Cazenave A., Berthier E, Meyssignac B., Le Cozannet G., Masson-Delmotte V., Salas y Mélia D., 2015. Le niveau de la mer : variations passées, présentes et futures. La Météorologie, 88, 69-82.

Ciais P., Sabine C., Bala G., Bopp L., Brovkin V., Canadell J., Chhabra A., DeFries R., Galloway J., Heimann M., Jones C., Le Quéré C., Myneni R.B., Piao S., Thornton P., 2013. Carbon and other biogeochemical cycles. In: Climate Change 2013: The Physical Science Basis. Contribution of Working Group I to the Fifth Assessment Report of the Intergovernmental Panel on Climate Change (Stocker T.F., Qin D., Plattner G.-K., Tignor M., Allen S.K., Boschung J., Nauels A., Xia Y., Bex V., Midgley P.M., eds). Cambridge University Press, Cambridge, UK, New York, NY, USA.

Collins M., Knutti R., Arblaster J., Dufresne J.-L., Fichefet T., Friedlingstein P., Gao X., Gutowski W.J., Johns T., Krinner G., Shongwe M., Tebaldi C., Weaver A.J., Wehner M., 2013. Long-term climate change: projections, commitments and irreversibility. In: Climate Change 2013: The Physical Science Basis. Contribution of Working Group I to the Fifth Assessment Report of the Intergovernmental Panel on Climate Change (Stocker T.F., Qin D., Plattner G.-K., Tignor M., Allen S.K., Boschung J., Nauels A., Xia Y., Bex V., Midgley P.M., eds). Cambridge University Press, Cambridge, UK, New York, NY, USA.

Douville H., Ribes A., Decharme B., Alkama R., Sheffield J., 2013. Anthropogenic influence on multidecadal changes in reconstructed global evapotranspiration. Nat. Clim. Change, 3, 59-62. doi:10.1038/nclimate1632

Dufresne J.-L., Bony S., 2008. An assessment of the primary sources of spread of global warming estimates from coupled atmosphere-ocean models. J. Climate, 21, 51355144.

Dufresne J.-L., Salas y Mélia, D., Denvil S., Tyteca S., Arzel O., Bony S., Braconnot P., Brockmann P., Cadule P., Caubel A., Chauvin F., Déqué M., Douville H., Fairhead L., Fichefet T., Foujols M.-A., Friedlingstein P., Gueremy J.-F., Hourdin F., Idelkadi A., Levy C., Madec G., Marquet P., Marti O., Musat I., Planton S., Royer J.-F., Swingedow D., Voldoire A., 2006. Simulation de l'évolution récente et future du climat par les modèles du CNRM et de I'IPSL. La Météorologie, 55, 45-59.

Flato G., Marotzke J., Abiodun B., Braconnot P., Chou S.C., Collins W., Cox P., Driouech F., Emori S., Eyring V., Forest C., Gleckler P., Guilyardi E., Jakob C., Kattsov V., Reason C., Rummukainen M., 2013. Evaluation of climate models. In: Climate Change 2013: The Physical Science Basis. Contribution of Working Group I to the Fifth Assessment Report of the Intergovernmental Panel on Climate Change (Stocker T.F., Qin D., Plattner G.-K., Tignor M., Allen S.K., Boschung J., Nauels A., Xia Y., Bex V., Midgley P.M., eds). Cambridge University Press, Cambridge, UK, New York, NY, USA.

Gedney N., Huntingford C., Weedon G.P., Bellouin N., Boucher O., Cox P.M., 2014. Detection of solar dimming and brightening effects on Northern Hemisphere river flow. Nat. Geosci., 7, 796-800. doi:10.1038/nge02263

Geoffroy 0., Saint-Martin D., Voldoire A., Salas y Mélia D., Sénési S., 2014. Adjusted radiative forcing and global radiative feedbacks in CNRM-CM5, a closure of the partial decomposition. Clim. Dyn., 42, 1807-1818. doi:10.1007/s00382-013-1741-9

Guivarch C., Cassen C., 2015. L'atténuation du changement climatique : retour sur le 5e rapport du Giec. La Météorologie, 88, 97-105.

Kharin V.V., Zwiers F.W., Zhang X., Wehner M., 2013. Changes in temperature and precipitation extremes in the CMIP5 ensemble. Clim. Change, 119, $345-357$.

Kirtman B., Power S.B., Adedoyin J.A., Boer G.J., Bojariu R., Camilloni I., Doblas-Reyes F.J., Fiore A.M., Kimoto M., Meehl G.A., Prather M., Sarr A., Schär C., Sutton R., van Oldenborgh G.J., Vecchi G., Wang H.J., 2013. Near-term climate change: projections and predictability. In: Climate Change 2013: The Physical Science Basis. Contribution of Working Group I to the Fifth Assessment Report of the Intergovernmental Panel on Climate Change (Stocker T.F., Qin D., Plattner G.-K., Tignor M., Allen S.K., Boschung J., Nauels A., Xia Y., Bex V., Midgley P.M., eds). Cambridge University Press, Cambridge, UK, New York, NY, USA.

Khodri M., Swingedouw D., Mignot J., Sicre M.A., Garnier E., Masson-Delmotte V., Ribes A., Terray L., 2015. Le climat du dernier millénaire. La Météorologie, 88, $36-47$.

Kodra E., Steinhaeuser K., Ganguly A.R., 2011. Persisting cold extremes under 21st-century warming scenarios. Geophys. Res. Lett, 38, L08705. doi: 10.1029/2011GL047103

Kravitz B., Robock A., Stenchikov G., Taylor K., Boucher 0., Schmidt H., Schulz M., 2011. The Geoengineeering Model Intercomparison Project (GeoMIP). Atmos. Sci. Lett., 12, 162-167. doi:10.1002/asl.316

Masson-Delmotte V., Braconnot P., Kageyama M., Sepulchre P., 2015. Qu'apprend-on des grands changements climatiques passés ? La Météorologie, 88, 25-35.

Massonnet F., Fichefet T., Goosse H., Bitz C.M., Philippon-Berthier G., Holland M.M., Barriat P.-Y., 2012. Constraining projections of summer Arctic sea ice. The Cryosphere, 6 , 1383-1394.

Meehl G.A., Tebaldi C., Walton G., Easterling D., McDaniel L., 2009. Relative increase of record high maximum temperatures compared to record low minimum temperatures in the US. Geophys. Res. Lett., 36, L23701. doi: 10.1029/2009GL040736 
Moss R.H. et al., 2010. The next generation of scenarios for climate change research and assessment. Nature, 463, 747-756.

O'Gorman P., 2012. Sensitivity of tropical precipitation extremes to climate change. Nat. Geosci., 5, 697-700. doi:10.1038/ngeo1568

0'Gorman P., Allan R.P., Byrne M.P., Previdi M., 2012. Energetic constraints on precipitation under climate change. Surv. Geophys., 33, 585-608.

O'Gorman P., 2014. Contrasting responses of mean and extreme snowfall to climate change. Nature, 512, 416-418.

Planton S., Bopp L., Brun É., Cattiaux J., Chevallier M., Ciais P., Douville H., Giraud G., Soubeyroux J.-M., Terray L., 2015. Évolution du climat depuis 1850. La Météorologie, 88, 48-55.

Risi C., 2013. L'augmentation du $\mathrm{CO}_{2}$ agit directement sur les précipitations. La Météorologie, 82, 3.

Schoetter R., Cattiaux J., Douville H., 2014. Changes of western European heat wave characteristics projected by the CMIP5 ensemble. Clim. Dyn. doi:10.1007/s00382-0142434-8

Sillmann J., Kharin V.V., Zwiers F.W., Zhang X., Bronaugh D., 2013. Climate extremes indices in the CMIP5 multimodel ensemble: Part 2. Future climate projections. J. Geophys. Res. Atmos., 118, 2473-2493.

Taylor K.E., Stouffer R.J., Meehl G.A., 2012. An overview of CMIP5 and the experiment design. Bull. Am. Meteorol. Soc., 93, 485-498.

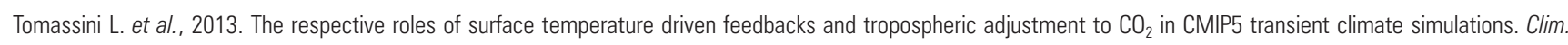
Dyn., 41, 3103-3126. doi:10.1007/s00382-013-1682-3

Trenberth K., 2011. Changes in precipitation with climate change. Clim. Res., 47, 123-138. doi:10.3354/cr00953

van Vuuren D.P., Edmonds J., Kainuma M., Riahi K., Thomson A., Hibbard K., Hurtt G.C., Kram T., Krey V., Lamarque J.-F., Masui T., Meinshausen M., Nakicenovic N., Smith S.J., Rose S.K., 2011. The representative concentration pathways: an overview. Clim. Change, 109, 5-31.

Vial J., Dufresne J.-L., Bony S., 2013. On the interpretation of inter-model spread in CMIP5 climate sensitivity estimates. Clim. Dyn., 41, 3339-3362. 